\title{
La identidad censurada: representación y manipulación de la homosexualidad en la obra Té y simpatía
}

\author{
Antonio J. Martínez Pleguezuelos \\ y J. David González-Iglesias González \\ Universidad de Salamanca \\ ajmp@usal.es; forloyo@usal.es
}

\begin{abstract}
Resumen: El objetivo de este artículo es analizar las consecuencias de la acción censora en la creación de la identidad homosexual, para lo cual llevamos a cabo un estudio contrastivo inglés-español de la obra de teatro estadounidense Tea and Sympathy (1953) de Robert Anderson y su adaptación al cine en 1956. En dicho análisis partiremos de la idea de censura presentado por Butler para examinar la selección léxica sexualmente marcada en ambas lenguas, las omisiones, las estructuras semánticas y sintácticas e incluso los cambios en la trama que introdujo la censura para comprobar cómo se recrea la realidad y qué asimetrías se producen en el proceso traductor en función de los sistemas simbólicos heterocéntricos imperantes en el contexto histórico.
\end{abstract}

Palabras clave: censura; identidad; homosexualidad; Té y simpatía.

\begin{abstract}
This article analyzes the consequences of the acts of censorship on the representation of homosexual identity. To do so, we carry out an EnglishSpanish contrastive study of the American play Tea and Sympathy (1953), by Robert Anderson, and its cinema adaptation in 1956. We will start from the concept of "censorship" which Butler presents to examine the sexually connoted lexical selection that has been chosen for both languages, together with the omissions, the semantic and syntactic structures and even the plot alterations applied by censors in order to assess the representation of reality and the asymmetries that are created in the process of translation according to the heterocentric symbolic systems that prevailed at the historical context.
\end{abstract}

Keywords: censorship; identity; homosexuality; Tea and Sympathy.

\) Martínez Pleguezuelos, Antonio J. \& González-Iglesias González, J. David. 2015. "La identidad censurada: representación y manipulación de la homosexualidad en la obra Té y simpatía". Quaderns de Filologia: Estudis Literaris XX: 53-67. doi: $10.7203 /$ qdfed.20.7528 

To the extent that homosexual attachments remain unacknowledged within normative heterosexuality, they are not merely constituted as desires that emerge and subsequently become prohibited. Rather, these are desires that are proscribed from the start. And when they do emerge on the far side of the censor, they may well carry that mark of impossibility with them, performing, as it were, as the impossible within the possible.

Judith Butler (1993: 25)

\section{La censura como construcción discursiva}

En el diccionario de cultura gay y lésbica Para entendernos de Alberto Mira, el autor señala en la entrada de "censura" sobre la homosexualidad que, a lo largo del tiempo,

el borrado de su existencia y el silencio son culturalmente elementos definitorios de la homosexualidad y siguen siendo la causa de que parezca a los no entendidos algo oscuro, oculto o pecaminoso (Mira, 2002: 169).

Los distintos modos de censura, continúa este autor, eliminan tanto aquellos movimientos que se identifican con la causa homosexual como la propia representación de la homosexualidad en las distintas manifestaciones artísticas, literarias o informativas. Debido a que solo a través de la representación de la identidad homosexual es posible percibirla como una entidad social (Cameron y Kulick, 2004: 12), si se produce una exclusión en cualquier medio comunicativo, aunque sea a través de mecanismos sutiles e imperceptibles, el discurso en torno a la homosexualidad, entendido en clave foucaultdiana, quedará relegado a un plano social invisible ${ }^{1}$.

Tomando como punto de partida teórico el trabajo de Foucault tanto en torno al discurso del poder (1979) como sobre la constitución de la sexualidad a través del discurso que la moldea y el lenguaje mediante el cual los discursos circulan (Foucault, 1989), Judith Butler reinterpretó

\footnotetext{
${ }^{1}$ En una sociedad como la nuestra son bien conocidos los procedimientos de exclusión. El más evidente [...] es lo prohibido. Uno sabe que no tiene derecho a decirlo todo, que no se puede hablar de todo en cualquier circunstancia, [...]. He ahí el juego de [...] prohibiciones que se cruzan, se refuerzan o se compensan, formando una compleja malla que no cesa de modificarse (Foucault, 2011: 14-15).
} 
la obra foucaultdiana para estudiar el juego de poderes que luchan por imponerse a la hora de reconstruir la figura de gays y lesbianas que se entretejen en la compleja malla discursiva del poder (Butler, 1990). El fruto de los trabajos de Butler dio como resultado una relectura de las teorías postestructuralistas sobre el lenguaje que aplicó en el terreno de la sexualidad para desvelar que el discurso que rige este ámbito en las sociedades actuales se ha convertido en un instrumento de producción y autorreproducción de poder que regula las identidades (Butler, 2007: 93-94; para un estudio en profundidad sobre esta línea teórica de Butler véase Pérez Navarro, 2005). El poder del discurso al que hace referencia la filósofa le llevó a cuestionarse si la vulnerabilidad lingüística del sujeto frente al lenguaje podría ser una consecuencia de su constitución lingüística:

Si estamos formados en el lenguaje, entonces este poder constitutivo precede y condiciona cualquier decisión que pudiéramos tomar sobre él, insultándonos desde principio, desde su poder previo (Butler, 2009: 16).

A partir de esta tesis, en una de sus líneas de investigación, Butler revisó el sentido tradicional del concepto censura, concebido como una privación de la libertad de expresión aplicada contra las personas o contra el discurso específico que desarrollan. Según esta acepción, de manera habitual se ha asumido que cualquier acto censor supone una restricción del habla que condiciona lo que es apropiado y posible en un determinado contexto. No obstante, Butler apuntó en su investigación hacia una nueva concepción de censura en tanto que forma de producción de habla que limita, de manera preventiva, qué es aceptable o no en una situación concreta (2009: 213; para un comentario crítico al respecto véase también Salih, 2002).

Desde este punto de vista, la filósofa entiende la censura, bien sea aplicada institucionalmente, implantada desde una moralidad religiosa concreta o autoimpuesta, como una forma de producción discursiva que marca los límites de lo que se puede concebir en una sociedad:

Por "productivo" no entiendo positivo o beneficioso, sino una visión del poder como formador y constitutivo, esto es, no concebido exclusivamente como el ejercicio externo de un control o como la privación de las libertades (Butler, 2009: 218). 
En este desarrollo teórico, la censura no es solo un mecanismo que restringe y acota la voluntad de los sujetos para expresarse con libertad, sino que actúa configurando al propio sujeto y al conjunto de relaciones interpersonales que lo constituyen (Pérez Navarro, 2004). En este argumento Butler apunta que esta idea de censura precede al discurso, al propio texto, y para hacer que este sea inteligible debe desarrollarse "a través de un proceso de selección que excluye algunas posibilidades y que escoge otras" (Butler, 2009: 213). De ahí la necesidad de entender la censura en sentido amplio como constructora del sujeto que habla y de observar la pertinencia de llevar a cabo un análisis de las presuposiciones discursivas de las que dependen los criterios constitutivos de la identidad.

Para profundizar en esta idea y comprobar la relevancia de este razonamiento teórico a la hora de recrear identidades sexuales que escapan del marbete de la heterosexualidad pasamos a analizar en el siguiente punto la obra Tea and Sympathy de Robert Anderson, traducida al español por María Luz Regás en 1955, pues en el proceso de reescritura descubriremos que es fácil distinguir cómo se moldea la identidad sexual tanto a través del discurso explícito como de todo aquello que no se puede contar (Santaemilia, 2008).

\section{Análisis contrastivo inglés-español de Tea and Sympathy}

La trama de esta obra teatral gira en torno a Tom Lee, un joven de casi 18 años con una gran sensibilidad, que cursa su primer año de universidad. Vive durante el curso escolar junto a otros compañeros en casa de un profesor, Bill, y su esposa Laura. El argumento comienza cuando se desvela que Tom ha estado en la playa bañándose y tomando el sol con un profesor, del que se sospecha que es homosexual. De esta forma se extiende la idea de que Tom es gay, aunque él está secretamente enamorado de Laura. Finalmente, después de ser acosado por compañeros y profesores por tal rumor, la obra acaba cuando Tom y Laura mantienen una relación sexual.

En función del argumento teórico defendido por Butler sobre la censura que adoptamos en esta investigación, el proceso de selección de las posibilidades lingüísticas que hacen que un texto sea inteligible presupone una toma de decisiones por parte del autor (o del traductor). No obstante, continúa la filósofa, no es el autor, ni el traductor, el que marca las normas por las que se realiza dicha selección, "están decididas 
con anterioridad a cualquier decisión individual" (Butler, 2009: 214) por el propio contexto donde se desarrolla el discurso. Este intento por purificar el ámbito público a través de la institucionalización de unas normas tácitas supone una censura preventiva (ibid.) por el miedo a la contaminación y al desarrollo de formas no deseadas por instituciones y órganos de poder. Como muestra, en el caso de nuestro estudio el temor a la censura del aparato franquista en la España de la dictadura hacía que el traductor se (auto)impusiera unos límites a la hora de tratar la sexualidad y, especialmente, la homosexualidad.

Por estos motivos, la traducción de esta obra para su posterior interpretación en el teatro español estuvo influida por la percepción negativa de la homosexualidad en el mundo hispanohablante en general, y por la dura censura franquista que vivía España en particular, lo que afectó de manera general a las traducciones realizadas en aquel período (Merino, 2008). Por ello, como pasamos a comprobar a través de distintos ejemplos extraídos de la traducción, muchas referencias al contenido sexual, y sobre todo a la homosexualidad, quedan veladas, matizadas, connotadas negativamente e incluso eliminadas (Pérez López de Heredia, 2004: 179-184).

En una obra como la que nos ocupa, que comenzó a dejar entrever un nuevo paradigma de masculinidad en el que ya no solo tiene cabida el ideal de hombre como sinónimo de fuerza y poder, resultarán especialmente relevantes los mecanismos discursivos empleados a la hora de recrear tanto la heterosexualidad como la homosexualidad. Así, en nuestro análisis estaremos igualmente atentos al conjunto de masculinidades hetero y homosexuales marcadas por contextos históricos, geográficos, culturales y sexuales que aparecen en el original y a cómo se han llevado al lector y espectador español. En este proceso creador de identidades la traducción desempeña un papel fundamental por su función de espejo capaz de reflejar otra cultura y por el poder que presenta a la hora de fijar conocimiento y estereotipos en torno a la identidad o, por el contrario, descubrir nuevos horizontes y nuevas vías de concebir la realidad y al sujeto (Vidal Claramonte, 2007).

\subsection{Análisis léxico}

Ya en las primeras páginas de Tea and Sympathy podemos encontrar una opción léxica de traducción muy significativa para nuestro estudio. En 
un diálogo de Laura con una de sus amigas el guion dice: "What all the boys in this school are thinking about. Not only now in the Spring, but all the time -Sex!”, que se tradujo al español como: “¿En qué piensan él y todos los muchachos de este colegio? Y no solamente en primavera, ¿eh? Siempre... Piensan en el pecado original" [nuestro subrayado]. Observamos así que en la versión española se huye de la explicitación del contenido sexual y que la elección léxica adopta un tinte entre lo religioso y lo moral que se aleja del original y comienza a ofrecernos un punto de vista diferente. En esta misma línea, si avanzamos un poco más en la obra, encontramos un nuevo ejemplo muy revelador para nuestro trabajo. En una de las escenas en la que los compañeros de universidad invaden la habitación de Tom para ver desde su ventana cómo una vecina le da el pecho a su hijo, uno de ellos se dirige al protagonista y le dice: “ [...] Or aren't you interested in women?", traducido como: "¿O es que no te interesan esos paisajes?" [nuestro subrayado]. Comprobamos en este caso que la traductora intenta que pase inadvertido el acento que pone el original en señalar el escaso interés de Tom por las mujeres, condicionado por la atenta mirada censora que imponía el régimen franquista. Sin embargo, este proceso de recreación de la figura del homosexual no se limita a encubrir o disimular las tendencias sexuales que, según los rumores, tiene el protagonista. Más allá, en una relectura atenta de la versión en español, percibimos que la selección léxica recrea un discurso marcado ideológicamente en una dirección concreta. Se impone un proceso de exclusión que aleja al homosexual de lo comúnmente aceptado y lo marca como diferente, extraño e incluso enfermizo, siguiendo las imposiciones que el régimen franquista guardaba con celo. Como ejemplo de ello podemos señalar los siguientes casos extraídos de la comparación entre original y traducción:

1. "HERB: I know. I shouldn't ask you. I know. I don't believe Tom was really involved with this fellow": "Yo sí lo sé. Y no sé por qué te lo pregunto. Yo sí lo sé. Por supuesto, yo no creo que a Tom lo haya podido envolver ese canalla" [en referencia al profesor homosexual]. 2. "HERB: Yes. He's being fired because he's been doing a lot of suspicious things around apparently, and this finished it. All right, I'll say it plain, Tom. He's a fairy. A homosexual": "Acaban de destituirlo por su conducta sospechosa. Este asunto ha colmado la medida. En una palabra, te lo diré claramente, Tom: es un anormal". 
3. "BILL: And you're doing this -all because of this- this fairy?": "Y todo eso por anormal".

4. "BILL: You were more interested in mothering that fairy up there than in being my wife": "¿Quieres decirme por qué te interesa más cuidar de un ser enfermizo que ser mi mujer?"

Según los ejemplos anteriores y retomando las palabras de Butler, la esfera comunicativa del lenguaje fija un campo de obscenidad que intenta excluir, siempre de manera parcial, de su misma operación (Butler, 2009: 214). De esta forma, las condiciones restrictivas que impone el contexto en el cual se desarrolla el discurso limitan las posibilidades lingüísticas, lo que establece aquello que debería incluirse adecuadamente en función de la "censura preventiva" (ibíd.) que marca la situación comunicativa. Esta forma implícita de censura reforzará los límites de lo enunciable y constituye la norma de lo que debe ser el sujeto y su sexualidad, rechazando de pleno, en el contexto de nuestro estudio, la homosexualidad. Con ello descubrimos que, efectivamente, los mecanismos censores no solo están involucrados en la construcción del sujeto, sino que "circunscribe[n] los parámetros sociales de lo que se puede decir en el discurso, de qué será admisible o no en el discurso público" (Butler, 2009: 218).

De forma paralela, hemos de recordar que el personaje de Tom no es gay, por lo que a través de estos dispositivos tácitos de censura no solo vemos un proceso de estigmatización de la homosexualidad, sino que además se fija y constituye al mismo tiempo el estereotipo de hombre rudo sin sensibilidad. Como señalábamos anteriormente, a través de los límites que marca el discurso, se construye y se establece una única identidad masculina posible frente a la pluralidad de masculinidades posibles.

\subsection{Análisis de cambios en el contenido}

Además de la terminología empleada en la versión española, resultan especialmente llamativos algunos cambios y reestructuraciones en las reformulaciones que utiliza la traductora para perder o difuminar las alusiones al supuesto comportamiento homosexual del protagonista. Algunos de los ejemplos más destacados extraídos de nuestro análisis son los siguientes: 
1. [En la escena en que espían a la vecina dando el pecho a su hijo, uno de los compañeros dice de Tom] "RALPH: [...] Maybe if it was Dr. Morrison instead of Mrs. Morrison, he'd be more interested": "A ti no te interesan estas cosas".

2. "RALPH: At least I'm normal. I like to look at pictures of naked girls -not men, the way Tom does": "Por lo menos soy normal. Me gustan las fotos de chicas desnudas; me gusta todo lo que no le gusta a Tom". 3. [Cuando Laura discute con su marido por todo el asunto de Tom] "LAURA: The facts! What facts! An innocent boy goes swimming with an instructor -an instructor whom he likes because this instructor is one of the few who encourage him, who don't ride him. -And because he's an off-horse, you and the rest are only too glad to put two and two together and get a false answer": “ $i L o s$ hechos! ¿Qué hechos? Un muchacho inocente va a nadar con un profesor que le es simpático, porque es de los pocos que lo animan, que no se burlan de él... Y porque ese muchacho es distinto a todos los demás, se complacen en hundirlo, manchándolo con la mala reputación del otro".

4. En la conversación entre el compañero de habitación de Tom y Laura, hablando sobre Tom "AL: (Almost whispering it) Well, he does act sort of queer, Mrs. Reynolds. He- LAURA: You never said this before. You never paid any attention before. What do you mean, "queer"? [...] AL: Well, like the fellows say, he sort of walks lightly, if you know what I mean. Sometimes the way he moves -the things he talks aboutlong-hair music all the time": "AL: (Casi en un susurro). -También él... es un poco raro, hay que reconocerlo, señora Reynolds. LAURA: Es la primera vez que se lo oigo decir. ¿Qué quiere decir usted con eso de $\operatorname{raro}$ ? [...] AL: No sé... dicen los muchachos que camina como... como pisando huevos... No sé si usted me entiende. Y a veces hace unos ademanes y habla de unas cosas. De música, horas y horas, hasta que a uno le crece la barba".

Precisamente este último ejemplo es una muestra clara de cómo la identidad homosexual queda diluida en la versión en español, pues aunque sepamos que el término queer en inglés pueda ser tanto 'raro' como 'gay', si tenemos en cuenta el conflicto del argumento, en la traducción se ha priorizado la idea de extraño y se marca la diferencia, con lo que se observa el intento de excluir a lo largo de la obra la condición homosexual y hacerla ajena al lector, con características que señalan de nuevo una identidad masculina preestablecida dominante frente al resto. De esta forma, tal y como apunta Judith Butler, estas prácticas discursivas excluyentes constituyen a los sujetos de un modo esencialista, 
según estructuras de poder anquilosadas e ideas preconcebidas que, en ningún caso, reconocen la pluralidad de sexualidades que existen en la realidad (Butler, 2006).

Esta visión de la censura como forma "productiva" que plantea Butler con la que iniciábamos estas páginas no considera las imposiciones censoras únicamente como el medio mediante el cual regular el habla. Más allá, y tal y como comprobamos en el tercer ejemplo que mostramos, los actos censores implican otros tipos de fines sociales como el fortalecimiento de concepciones de la legitimidad, del consenso y la autonomía cultural e histórica concretas:

En la versión más extrema de este tipo de instrumentalización, el habla es algo totalmente secundario respecto a los fines de la censura o, más bien, el habla funciona como una tapadera del objetivo político real de la censura, que tiene poco o nada que ver con el habla (Butler, 2009: 219).

De este modo, entendemos que la formación del sujeto no se realiza solo a través de la regulación del habla, sino por la "regulación del ámbito social del discurso enunciable" (ibid.). Desde este punto de vista, lo que se puede o no se puede decir resulta una cuestión secundaria frente a la relevancia que adquiere el ámbito dentro del cual se puede hablar y construir el discurso en torno a la homosexualidad. Por tanto, los sujetos que escapan de la frontera de la heterosexualidad, en tanto que sujetos lingüísticos, están condicionados por una serie de normas explícitas que limitan el tipo de habla.

La cuestión principal en este caso no es localizar la censura impuesta sobre las expresiones pronunciadas por un sujeto concreto, sino entender cómo una acción censora determina quién puede constituirse en sujeto en función de si el habla de ese individuo cumple las normas de los que se puede decir y lo que no (Butler, 2009: 220). En este caso, situarse fuera del campo de lo expresable supone un reto al discurso heterodesignado por poner en tela de juicio el discurso establecido, romper los límites de la censura y convertirse en un sujeto enunciable más allá de la etiqueta de la heterosexualidad. Esta sería claramente una muestra evidente del "discurso imposible" que presenta Butler por adentrarse en los laberintos identitarios de lo asocial que escapan de las identidades discursivas posibles $\mathrm{y}$, por tanto, permitidas. 
Por cuanto hemos dicho y según extraemos de los casos presentados, las imposiciones que influyen en la perspectiva de la traductora a la hora de abordar la traducción de la obra contrastan marcadamente con el objetivo del autor original, Anderson, cuyo objetivo era destruir la ecuación que equipara la heterosexualidad como única forma inteligible de identidad sexual. Además, en un paso posterior, reconocemos que la obra original supone un acto subversivo ante el ejercicio normativo del poder, ya que no relaciona inevitablemente la heterosexualidad con la idea de macho rudo que no muestra nunca sus sentimientos. En el lado opuesto, los ejemplos que hemos visto y que siguen a continuación son una muestra de que en español ha perdido gran parte de su carácter revolucionario y el efecto que consigue es diferente. Si bien la línea argumental es la misma, las estrategias de traducción siguen perpetuando estereotipos y continúan reforzando los binomios mujer-sumisión, hombre heterosexual-fuerza y poder, y homosexualidad-debilidad.

\subsection{Omisiones y adiciones discursivas}

Además de la selección léxica y las alteraciones a la hora de reformular la obra, resultan muy llamativas las adiciones o eliminaciones aplicadas sobre el original que añaden o roban información al espectador español y que continúan moldeando las identidades de los personajes. Estas operaciones tácitas de censura, en clave butleriana, son una muestra clara de cómo el discurso produce lingüísticamente los parámetros que conforman el sujeto. Como ejemplo, en el siguiente caso vemos que, mientras que en inglés no se dice nada, en español se inserta el siguiente comentario de uno de los estudiantes: "RALPH: ¿Qué pasa? STEVE: Ha pasado al nene al surtidor de la derecha" [nuestro subrayado] mientras la vecina da el pecho a su bebé, reafirmando la idea de necesidad de deseo (hetero)sexual hacia las mujeres. Más adelante se añade igualmente en la versión española la siguiente conversación completa mientras dos compañeros bajan una escalera: "-La gordita es bárbara. -¡Pero Rosy tiene un par de...ojos! -iQué ojos! ¡Las piernas son lo bueno!”

Se trata de diálogos que potencian la identidad heterosexual mediante la presentación de un hombre que puede y además debe desear a la mujer para reafirmar su posición y su identidad. La formación del sujeto, como venimos señalando, se lleva a cabo mediante la restric- 
ción del campo de lo que se puede decir, pero también a través del discurso performativo que se repite como elemento vertebrador de la identidad (Butler, 1990). De esta forma, con estas adiciones se limita y censura, por acción inversa, lo que debe constituir la identidad sexual y se potencian los rasgos del deseo heterosexual frente al ocultamiento del homosexual. En paralelo, el resultado de la no traducción de las partes señaladas en los siguientes ejemplos persigue los mismos objetivos:

1. "RALPH: Hell, how would you like to have to tell your wife, "Honey, I've been saving myself for you, except for one night when a guy...". 2. 'RALPH: Didn't you notice all those strong man poses he's got in his bottom drawer? AL: Yes, I've noticed them. His old man wants him to be a muscle man, and he wrote away for this course in muscle building -and they send those pictures. Any objections?".

3. "HERB: I'm drawing no conclusions. This sort of thing can happen to a normal boy. But it's what the others will think -Added to the Harris business. And that's all that's important", que queda como: "HERB: Sí, ya sé que eso le puede suceder a cualquier muchacho. Pero lo importante es lo que piensan los demás".

La normatividad del lenguaje heterodesignado, comprobamos en estos casos, posibilita que el sujeto se constituya dentro de los límites de lo expresable. Funcionar en este lugar del discurso permite la construcción del sujeto, por lo que expresarse fuera de este ámbito, de forma imposible, no se podrá considerar como el habla de un sujeto y su viabilidad será cuestionada por las normas tácitas de la censura que permean y rigen una sociedad. Este acto de exclusión y prohibición es el efecto reiterado de una estructura anterior: "algo ha sido excluido, pero ningún sujeto lo excluye; el sujeto aparece como un efecto de la propia exclusión" (Butler, 2009: 226). Dicha acción no se realiza exactamente sobre un sujeto previo al discurso, sino que, como indica Butler, se lleva a cabo de tal modo que el sujeto mismo resulta una producción performativa derivada del acto de prohibición (ibid.). Por tanto, podemos concluir que si el sujeto se crea a través de un conjunto de prohibiciones entendidas bajo el signo de la censura, la propia limitación constitutiva del sujeto marca el contexto en el cual dicho sujeto será posible. 


\section{La adaptación al cine de Vincente Minelli}

En cuanto a la adaptación a la gran pantalla de la obra de teatro y su posterior traducción al español, es necesario de nuevo recordar el contexto y los condicionantes que llevan aparejados. El Production Code Administration estadounidense, organismo encargado de velar por la moral de los ciudadanos, impuso serias restricciones al argumento y al desarrollo de la obra (Pérez López de Heredia, 2004: 184). Aunque en general la trama se mantiene, el desencadenante de los hechos deja de ser la escapada a la playa de Tom con su profesor y en la versión cinematográfica todo ocurre porque Tom pasa unos minutos cosiendo en la playa con algunas esposas de los profesores y es descubierto por sus compañeros. En ningún momento se emplean términos como queer o fairy en el original y tampoco se reproducen en español. Toda la trama en torno a la homosexualidad queda velada y no se habla claro en ningún momento de ella. El "problema" que se presenta al espectador se desplaza desde la supuesta homosexualidad a ser simplemente raro o diferente a los demás. Así, queda a la imaginación del espectador el imaginarse por qué Tom sufre el acoso de sus compañeros, aunque no será difícil apreciar que la diferencia entre el protagonista y el resto es su orientación sexual.

Desde el punto de vista traductológico, la comparativa de la versión original y la traducida ofrece datos mucho menos relevantes, en gran parte debido a que el original ya ha sufrido la censura estadounidense. Esto facilitó mucho el trabajo al censor español, que no encontró ninguna referencia clara al sexo ni a la homosexualidad, por lo que la traducción se limita a una reproducción fiel y cercana del original.

No obstante, cabe destacar que tanto la escasa presencia de la homosexualidad como el adulterio de Laura se abordan de una forma diferente, mucho más estricta, frente a la versión teatral. En un epílogo añadido a la versión cinematográfica, Laura escribe una carta de despedida a Tom que aclara, ante cualquier tipo de duda, que todos los pensamientos sobre una posible homosexualidad de éste, algo totalmente oculto en la película, son falsos. Además, como apunte llamativo, en esta versión Laura reconoce en esta carta su culpa por haber sido infiel a su marido, hecho que no ocurre en el original. En todo caso, el argumento y su traducción supone una extensión del argumento teórico sobre censura a la 
Butler que hemos recogido aquí como forma productora de identidades que restringe y establece la agencia del poder.

\section{Conclusiones}

A través de todos los ejemplos presentados hemos comprobado, tal y como vislumbrábamos desde las primeras páginas, que el lenguaje, y con él la traducción, crea discursos en torno a las distintas identidades de género y sexo con mecanismos que a simple vista pueden llegar a pasar inadvertidos para el lector. En concreto, la censura según la concibe Butler se convierte en un fuerza constructora de identidades previa al lenguaje. De acuerdo con las reglas de poder discursivas que rigen lo que se puede decir y lo que no, uno habla en función de un conjunto tácito de normas que permiten entender y concebir al sujeto. En esta situación, la censura actuará configurando los límites propios del discurso y, a la vez, al mismo sujeto.

Negar en este contexto la posibilidad de que existan otras identidades más allá de los límites de la heterosexualidad supondrá la exclusión y no percepción de todos aquellos sujetos cuya sexualidad se aleje de la heteronormatividad aceptada. De este modo, la reescritura censurada de cualquier texto se convertirá en una lectura interesada, cerrada al diálogo y que vuelve la espalda al carácter interseccional de cualquier realidad social.

Debemos, por ende, a partir del modelo presentado por Butler, mantenernos alerta en nuestra postura como receptores de textos y traducciones que vienen inevitablemente conformados por la censura en tanto que creadora del discurso. Sin duda, una tarea necesaria a la vista de la repercusión que pueden alcanzar los discursos traducidos a la hora de construir identidades a través de reescrituras interculturales.

\section{Referencias}

Butler, Judith. 1990. Gender Trouble. Nueva York/Londres: Routledge.

Butler, Judith. 1993. Critically queer. GLQ: A Journal of Gay and Lesbian Studies 1: 17-32.

Butler, Judith. [2004] 2006. Deshacer el género. Barcelona: Paidós. [Traducción de Patricia Soley-Beltrán]. 
Butler, Judith. [1999] 2007. El género en disputa. El feminismo y la subversión de la identidad. Barcelona: Paidós. [Traducción de M. Antonia Muñoz].

Butler, Judith. [1997] 2009. Lenguaje, poder e identidad. Madrid: Síntesis. [Traducción de Javier Sáez y Beatriz Preciado].

Cameron, Deborah \& Kulick, Don. 2004. Language and Sexuality. Cambridge: Cambridge University Press.

Foucault, Michel. 1979. Microfísica del poder. Madrid: La piqueta. [Traducción de Julia Varela y Fernando Álvarez-Uría].

Foucault, Michel. [1976] 1989. Historia de la sexualidad, 1. La voluntad del saber. Madrid: Siglo XXI. [Traducción de Ulises Guiñazú].

Foucault, Michel. [1970] 2011. El orden del discurso. Barcelona: Fábula. [Traducción de Alberto González Troyano].

Merino Álvarez, Raquel. 2008. La homosexualidad censurada: estudio sobre corpus de teatro TRACEti inglés-español (desde 1960). En Merino Ávarez, Raquel (ed.) Traducción y censura en España (1939-1985). Estudios sobre corpus TRACE: cine, narrativa, teatro. Bilbao: Universidad del País Vasco, 243-286.

Mira, Alberto. 2002. Para entendernos: diccionario de cultura homosexual, gay y lésbica. Barcelona: La tempestad.

Pérez López de Heredia, María. 2004. Traducciones censuradas de teatro Norteamericano en la España de Franco (tesis doctoral). Bilbao: UPV/ EHU.

Pérez Navarro, Pablo. 2004. Performatividad y subversión de la identidad: a propósito de la obra de Judith Butler. Laguna. Revista de filosofía 14: 147-164.

Pérez Navarro, Pablo. 2005. Cuerpo y discurso en la obra de Judith Butler: políticas de lo abyecto. En Córdoba, David; Sáez, Javier \& Vidarte, Paco (ed.) Teoría queer. Políticas bolleras, maricas, trans, mestizas. Barcelona/Madrid: Egales, 133-148.

Salih, Sara. 2002. Judtih Butler. Londres/Nueva York: Routledge.

Santaemilia, José. 2008. The Translation of Sex/Related Language: The Danger(s) of Self-Censorship(s). La formation en traduction: pédagogie, docimologie et technologie. Translation Training: Pedagogy, Evaluation and Technologies 21(2): 221-252.

Vidal Claramonte, M. C. África. 2007. Traducir entre culturas: poderes, diferencias, identidades. Frankfurt am Main: Peter Lang. 
